\title{
Caractérisation de quelques provenances de Pinus halepensis Mill. sur la base de la structure anatomique et morphologique des aiguilles
}

\author{
R. CALAMASSI \\ Département de Biologie végétale, Laboratoire de Botanique agricole et forestière \\ Ple delle Cascine 28,50144 Florence, Italie
}

\section{Résumé}

Quelques caractères anatomiques et morphologiques des aiguilles des jeunes plantes et des arbres adultes de Pinus halepensis Mill. provenant de 10 origines différentes, ont été examinés et analysés dans le but de caractériser les provenances et de vérifier la valeur discriminante de certains caractères en fonction de l'âge et du milieu de croissance.

Les résultats confirment la considérable variabilité de quelques caractères en fonction de l'âge des plantes et du milieu, et montrent que les caractères qui distinguent les provenances chez les jeunes plantes ne sont pas les mêmes que ceux qui les caractérisent à l'âge adulte.

La stabilité de certains caractères et leur association en quelques populations ont toutefois permis de distinguer, parmi les provenances étudiées, des groupements bien différenciés, représentés par les provenances grecques (A 2, A 3, A 4), par les provenances plus occidentales et/ou d'altitude (A 17, A 25, A 29, A 30), tandis que les provenances d'Italie centre-méridionale (A 26, A 27, A 28) se placent en position intermédiaire entre les deux groupes.

Une variation géographique, allant d'ouest à cst, a été mise également en évidence.

\section{Introduction}

L’aire naturelle de Pinus halepensis Mill. est assez étendue (NAHAL, 1962 ; Panetsos, 1981), et, selon Francini (1953), cette espèce est pourvue d'une ample capacité d'adaptation au milieu. Cela fait supposer l'existence d'écotypes à l'allure assez caractérisée, dont quelques-uns ont déjà été individualisés dans quelques provenances (Palmberg, 1975; Pelizzo \& Tocci, 1978).

La caractérisation des provenances de Pinus halepensis Mill. esi un des objectifs du Projet FAO 4 bis «Expériences internationales sur les provenances de Pinus halepensis et Pinus brutia», dont l'Institut Expérimental pour la Sylviculture d'Arezzo, est le coordinateur, et le présent travail a été entrepris dans le même but, 
ainsi que d'autres recherches déjà parues sur les caractères et les comportements des semences et des jeunes plantes (Pelizzo \& Tocci, 1978), par rapport à la germination (CAlamassi, 1982 ; Calamassi et al., 1984), et à la résistance aux stress des semences et des plantes adultes (Calamassı et al., 1980 ; Falusi et al., 1983 ; Falusi et al., 1984).

La caractérisation des provenances à partir de la morphologie et de l'anatomie est la première façon d'approcher le problème; et les données de la littérature établissent la crédibilité de cette méthode.

JäRIG (1962) classe les pins sur cette base, et l'identification de la plupart des espèces a recours à des clefs fondées sur les caractéristiques des feuilles (Durrell, 1916; Hosie, 1969). La structure anatomique des aiguilles peut être utilisée pour étudier la variabilité d'une espèce dans son milieu naturel, VIDAKovic (1957) classe les populations de Pinus nigra de Yougoslavie uniquement suivant cette méthode. Gellini (1968) éclaircit la position systématique du Pin noir de Villetta Barrea sur la base des couches de cellules de l'hypoderme et de la longueur de l'aiguille soit sur des individus dans leur milieu naturel, soit sur certains autres qui ont été élevés dans le même milieu (pépinière). Quelques autres auteurs ont utilisé cette méthode sur des plantes élevées dans le même milieu pour vérifier l'attribution des provenances à des sous-espèces (Arbez \& Millier, 1971), ou bien pour augmenter les connaissances sur les mécanismes de variation physiologique et génétique d'une espèce (Mergen, 1963). De même Vidakovic (1958), Keng \& Little (1961), Ferré (1965), NAPPZINN (1966), Roller (1966) soutiennent la valeur taxinomique des caractères anatomiques des aiguilles, pourvu que le matériel à étudier soit comparable.

Récemment Panetsos (1975) et Riva \& Vendramin (1983) ont fait recours à cette technique pour discriminer les hybrides (naturels et artificiels) de $P$, brutia $\times P$. halepensis.

Des recherches très détaillées ont été conduites pour évaluer les effets du milieu sur les caractères anatomiques des aiguilles (LEYTOON \& JUNIPER, 1963; LeYTON \& Armitage, 1968 ; Evans \& Millier, 1976; Baig \& Tranouillini, 1976); selon OPPENHEIMER (1968) les critères anatomiques et histologiques seuls ne suffisent pas pour caractériser exactement la résistance à la sécheresse, mais ils sont parfois de quelque intérêt pour en éclaircir plusieurs aspects.

Les descriptions des caractères anatomiques et morphologiques des aiguilles de Pinus halepensis Mill. peuvent avoir une valeur générale (Flora Europaea, 1964 ; Mirov, 1967), ou bien elles sont plus orientées, avec l'emploi de peu de critères, sur l’identification des hybrides (PAnetsos, 1975 ; Riva \& Vendramin, 1983). Colaone seul (1971) s'attache à l'identification des provenances de $P$. halepensis, élevées en pépinière, sur la base de la structure, mais il n'utilise qu'un nombre très limité de caractères et de provenances.

Lc but de cette recherche effectuéc sur 10 provenances de Pinus halepensis Mill. (tabl. 1), est la caractérisation de celles-ci sur une base anatomique et morphologique; de plus, la vérification de la valeur discriminante de quelques-uns des caractères examinés, leur stabilité ou variation en fonction de l'âge et, si possible, la comparaison de nos résultats avec ceux qui ont été obtenus dans les régions d'origine pour évaluer les effets du milieu de croissance. 


\section{TABLeaU 1}

Liste des provenances de Pinus halepensis Mill.

List of Pinus halepensis Mill. provenances.

\begin{tabular}{lcccc}
\hline Provenances & & Lat. Nord & Longitude & Altitude \\
\hline A2 Elée & Grece & $37^{\circ} 46^{\prime}$ & $21^{\circ} 32^{\prime} \mathrm{E}$ & $200 \mathrm{~m}$ \\
A3 Eubée & $"$ & $38^{\circ} 58^{\prime}$ & $23^{\circ} 18^{\prime} \mathrm{E}$ & $200 \mathrm{~m}$ \\
A4 Chalkidike I & $"$ & $40^{\circ} 1^{\prime}$ & $23^{\circ} 21^{\prime} \mathrm{E}$ & $125 \mathrm{~m}$ \\
A17 Guadalmedina & Espagne & $37^{\circ} 02^{\prime}$ & $2^{\circ} 15^{\prime} \mathrm{O}$ & $1000 \mathrm{~m}$ \\
A25 Imperia & Italie & $43^{\circ} 54^{\prime}$ & $8^{\circ} 03^{\prime} \mathrm{E}$ & $200 \mathrm{~m}$ \\
A26 Otricoli & $\prime \prime$ & $42^{\circ} 24^{\prime}$ & $12^{\circ} 38^{\prime} \mathrm{E}$ & $400 \mathrm{~m}$ \\
A27 Patemisco & $"$ & $40^{\circ} 39^{\prime}$ & $17^{\circ} 20^{\prime} \mathrm{E}$ & $5 \mathrm{~m}$ \\
A28 Vico del Gargano & " & $41^{\circ} 54^{\prime}$ & $16^{\circ} 00^{\prime} \mathrm{E}$ & $225 \mathrm{~m}$ \\
A29 Aures Beni Mejloul & Algerie & $35^{\circ} 10^{\prime}$ & $6^{\circ} 50^{\prime} \mathrm{E}$ & $1350 \mathrm{~m}$ \\
A30 Senalba & " & $34^{\circ} 40^{\prime}$ & $3^{\circ} 02^{\prime} \mathrm{E}$ & $1250 \mathrm{~m}$ \\
\hline
\end{tabular}

\section{Matériel et méthơles}

A la fin de la saison de croissance 1980 , sur 10 plantes de chaque provenance, ont été récoltés 20 brachyblastes appartenant à 2 branches différentes, sur la pousse de l'année et sur le côté supéricur de la cime. Les plantes étaient âgées de 4 ans, ayant été transplantées en 1977 dans la pépinière de l'Institut Expérimental pour la Sylviculture d'A rezzo. Dispositif constitué de 24 provenances $(10 \mathrm{P}$. halepensis, $13 P$. brutia, $1 P$. eldarica) selon schéma à bloc randomisé avec 30 répétitions.

Tous les brachyblastes ont été récoltés dans la même journée et réfrigérés jusqu'au moment de l'analyse.

Une opération similaire a été accomplie à la fin de saison de croissance 1984, lorsque toutes les provenances en étaient à la phase de reproduction (arbres adultes).

Une aiguille pour chaque brachyblaste a été examinée (200 aiguilles par provenances).

La longueur de l'aiguille et de la gaine a été mesurée au $\mathrm{mm}$ près. Dans la zone moyenne les lignes stomatifères ont été comptées, aussi bien du côté dorsal que du côté ventral; sur les sections transversales les dimensions de l'aiguille (largeur et hauteur) ont été mesurées (à l'oculaire micrométrique), et le nombre et la position des canaux sécréteurs ont été enregistrés.

Sur les aiguilles de 1984 , outre ce qui a déjà été cité, on a relevé le nombre des stomates par $\mathrm{cm}$ linéaire sur deux lignes, l'épaisseur de la cuticule des cellules de l'épiderme, et le nombre des couches de l'hypoderme sur des sections transversales de $18 \mu$. 
Pour distinguer la cuticule de la paroi cellulaire cutinisée, les sections ont été immergées dans l'acide chromique $(10 \mathrm{p} .100)$ pendant 10 minutes, lavées à l'eau et placées dans une solution saturée de Soudan III pendant 1 heure, lavées à l'alcool à 50 p. 100 et montées en jelly comme indiqué par BAig \& Tranouillini (1976).

Les résultats ont été analysés selon l'analyse de la variance à 1 et 2 facteurs, et les comparaisons effectuées à l'aide du test des moindres différences significatives (MDS), au niveau $P=0,01$, sauf indication différente.

Une matrice de corrélation linéaire a été calculée, utilisant la latitude, la longitude et l'altitude des stations d'origine des semences et les valeurs de chaque caractère anatomique et morphologique des aiguilles.

\section{Résultats}

\subsection{Longueur des aiguilles}

Dans le cas de jeunes plantes les différences dues à ce caractère sont significatives. En particulier les aiguilles d'A 28 et d'A 29 sont plus longues que celles d'A 25 et d'A 27 (tabl. 2); ces différences vont disparaître dans le cas des arbres adultes (tabl. 3), d'où résulte pour ceux-ci un classement très différent.

TABLEAU 2

Caractéristiques morphologiques et anatomiques (valeurs moyennes) des aiguilles des plantes de 4 années des provenances de Pinus halepensis Mill.

Morphological and anatomical needle characteristics (average values) of the 4 year old seedlings of Pinus halepensis Mill. provenances.

\begin{tabular}{|c|c|c|c|c|c|c|c|c|c|c|c|c|}
\hline \multirow{2}{*}{ Provenances } & \multirow{2}{*}{$A 2$} & \multirow{2}{*}{$A 3$} & \multirow{2}{*}{ A4 } & \multirow{2}{*}{ A17 } & \multirow{2}{*}{$\wedge 25$} & \multirow{2}{*}{$A 26$} & \multirow{2}{*}{ A27 } & \multirow{2}{*}{$A 28$} & \multirow{2}{*}{129} & \multirow{2}{*}{130} & \multicolumn{2}{|c|}{ MDS } \\
\hline & & & & & & & & & & & 0.01 & 0,05 \\
\hline \multicolumn{13}{|l|}{ Dimensions algutilea: } \\
\hline 1ongueur (ms) & BA, 4 & 85.5 & 86.8 & 93,3 & 82,3 & 87,8 & 80.4 & 100,3 & 97.1 & 93,0 & 14,50 & 11,00 \\
\hline leraeur $\quad(\mu)$ & $\mathbf{6 4 5}$ & 774 & 371 & 818 & 832 & 770 & 827 & 841 & 790 & 808 & 73,22 & 55,72 \\
\hline havteur $(\mu)$ & 538 & 501 & 498 & 532 & 538 & 509 & 533 & 537 & 502 & 515 & n. & s. \\
\hline Longueur galne (man) & 5,8 & 5,7 & 5,4 & 6.1 & 6.9 & 6,6 & 5,9 & 6,5 & 7.0 & 6.3 & 0.89 & 0,68 \\
\hline \multicolumn{13}{|l|}{ Canoux gécreteur: } \\
\hline total & 2,04 & 0,74 & 1,17 & 2,53 & 2,46 & 1,79 & 1,29 & 2,30 & 2,85 & 2,24 & 1,11 & 0,83 \\
\hline \multicolumn{13}{|l|}{ en position medime: } \\
\hline * réls & 20.52 & 35,30 & 15,54 & 13,25 & 7.75 & 56.52 & 21.37 & 20,75 & 13,15 & 8.64 & & \\
\hline * tranoformes & 23,48 & 31,70 & 16,45 & 17,29 & 12,86 & 47.49 & 24,10 & 23,53 & 16,53 & 14,13 & 19,25 & 14,65 \\
\hline \multicolumn{13}{|l|}{ Lignes stomatiferes: } \\
\hline dorsales & 6.2 & 5,5 & 5,9 & 5,9 & 6,4 & 5,7 & 5,6 & 5,7 & 6,4 & 5,7 & \multicolumn{2}{|c|}{ n.s. } \\
\hline ventrales & 2,7 & 2,6 & 2,7 & 2,4 & 2,5 & 2,1 & 2.5 & 2,5 & 2,8 & 2,2 & 0,44 & 0,35 \\
\hline totales & 8.9 & 8,1 & 0.6 & 8,3 & 6.9 & 7,8 & 8,1 & 8,2 & 9,2 & 7.9 & n. & s. \\
\hline
\end{tabular}


D'après les données du tableau 4, aucune différence significative n'a été mise en évidence, ni parmi les populations, ni entre les deux groupes de plantes d'âge différent.

\subsection{Longueur de la gaine}

Pour ce caractère aussi, les différences qui paraissent significatives dans le cas des jeunes plantes (tabl. 2), (A 29, A 25 et A 26 ont leurs gaines plus longues par rapport aux provenances grecques A 2 , A 3 et A 4), ne se maintiennent pas lorsqu'on passe aux aiguilles des arbres adultes (tabl. 3).

\section{TABLEAU 3}

Caractéristiques morphologiques et anatomiques (valeurs moyennes) des aiguilles des plantes de 8 années des provenances de Pinus halepensis Mill.

Morphological and anatomical needle characteristics (average values) of the 8 year old seedlings of Pinus halepensis Mill. provenances.

\begin{tabular}{|c|c|c|c|c|c|c|c|c|c|c|c|c|}
\hline \multirow{2}{*}{ Provunnnces } & \multirow{2}{*}{$\mathbf{A 2}$} & \multirow{2}{*}{ A3 } & \multirow{2}{*}{14} & \multirow{2}{*}{ A17 } & \multirow{2}{*}{ A25 } & \multirow{2}{*}{ A26 } & \multirow{2}{*}{ A27 } & \multirow{2}{*}{$A 28$} & \multirow{2}{*}{ A29 } & \multirow{2}{*}{ A.30 } & \multicolumn{2}{|c|}{ mos } \\
\hline & & & & & & & & & & & 0,01 & $0,0:$ \\
\hline \multicolumn{13}{|l|}{ Dlmeneions aigullies: } \\
\hline longueur ( $\mathrm{mm}$ ) & 69,4 & 92,8 & 93,5 & 92.4 & 82,8 & 80,4 & 85,7 & 84,2 & 91,4 & 85,5 & \multicolumn{2}{|c|}{ n.s. } \\
\hline largour & 894 & 907 & 929 & 1072 & 1050 & 997 & 959 & 939 & 1058 & 1043 & 73,32 & 58,79 \\
\hline hauteur & 649 & 610 & 609 & 689 & 686 & 657 & 638 & 627 & 690 & 685 & 46,40 & 35,30 \\
\hline Longueur gaine (mal) & 7,6 & 7,3 & 7,4 & 8,1 & 8,6 & 8,1 & 7,5 & 7,9 & 8,4 & 8,3 & n. & \\
\hline \multicolumn{13}{|l|}{ Caneux Escréteurs: } \\
\hline totel & 2,24 & 0,80 & 1.94 & 5.44 & 5,87 & 4,12 & 2,35 & 3,42 & 5,34 & 5,16 & 2.06 & 1.57 \\
\hline \multicolumn{13}{|l|}{ en poeltion modiane: } \\
\hline$x$ rtels & 25,03 & 24,26 & 6,39 & 2.79 & 1,61 & 17,24 & 12,59 & 9,10 & 0,17 & 2.74 & & \\
\hline$x$ transformen & 26,26 & 24,95 & 20,16 & 7,76 & 4,63 & 21,19 & 15,76 & 14,32 & 0,75 & 7,69 & 16,61 & 12.64 \\
\hline \multicolumn{13}{|l|}{ Lignes etomatiferes: } \\
\hline dorenales & 7.5 & 6.8 & 7,3 & 8,3 & 0,5 & 7,3 & 7,6 & 7,0 & 8,1 & 7,9 & 0,80 & 0,61 \\
\hline ventrales & 3,0 & 3,3 & 3,4 & 3,1 & 2,8 & 2,9 & 3.2 & 3,0 & 3,2 & 2,8 & 0,38 & 0,29 \\
\hline totales & 10.5 & 10,1 & 10.7 & 11,4 & 11,3 & 10,2 & 10,8 & 10.0 & 11.3 & 10,7 & 0,99 & 0.26 \\
\hline Stomates par $\mathrm{cm}$ & 96.5 & 99,4 & 92,6 & 82.3 & 82,8 & 75,4 & 81,8 & 84,2 & 87,1 & 84,7 & 6,46 & 4,91 \\
\hline Ipiderme & 17.13 & 16,20 & 17,05 & 17.52 & 16,21 & 17,86 & 17,22 & 17,33 & 18,30 & 18,07 & \multicolumn{2}{|c|}{ n.t. } \\
\hline Cellules hypoderme & 2.2 & 1,9 & 1,9 & 2.1 & 2,0 & 2,0 & 1,8 & 1,9 & 2.0 & 2,0 & \multicolumn{2}{|c|}{ n.s. } \\
\hline Cuticule & 3,06 & 2,88 & 3.09 & 3.49 & 3,05 & 3.20 & 3,33 & 3,15 & 3.48 & 3.51 & 0,44 & 0.33 \\
\hline
\end{tabular}

Pour ce caractère, à la différence de ce qui se vérifie pour la longueur des aiguilles, une augmentation générale des dimensions de la gaine se manifeste avec l'âge (tabl. 4), cela ne détermine aucun changement sur la position des provenances si on les classe selon la longueur de la gaine. 


\section{TABLEAU 4}

Longueur moyenne des aiguilles (a) et de la gaine (b) en mm, $n^{\prime \prime}$ des canaux sécréteurs (c), p. 100 des canaux sécréteurs en position médiane (d), largeur (e) et hauteur (f) des aiguilles en micron, $n^{\prime \prime}$ des lignes stomatifères totales ( $g$ ) en plantes de 4 et 8 années des provenances de Pinus halepensis Mill.

Needle (a) and needle-sheath (b) mean lengths $(\mathrm{mm}), n^{n}$ of resin canals (c), p. 100 of resin canals in median position $(d)$, width $(e)$ and height $(f)$ of needles $(u)$, total number of stomatal rows $(g)$ of 4 and 8 year old plants of the Pinus halepensis Mill. provenances.

\begin{tabular}{|c|c|c|c|c|c|c|c|c|c|c|c|}
\hline Provenance: & $A 2$ & $\mathbf{A 3}$ & $\mathbf{M}$ & A17 & 125 & 126 & 127 & 128 & 129 & 130 & Moyennes \\
\hline 4 années & 84,4 & 85.5 & 06,7 & 93.3 & 22,3 & 87.8 & 80.4 & 100,3 & 97,1 & 93,0 & 89,1 \\
\hline 8 annies & 89,4 & 92.8 & 93,5 & 92,4 & $B 2, B$ & 80,4 & 85.7 & 64,2 & 91,4 & 85,5 & 87,8 \\
\hline Moyernes & 86,9 & 89.2 & 90,1 & 92,8 & $\mathrm{~B} 2,8$ & 84.1 & 83,0 & 92,2 & 94.2 & 89,2 & \\
\hline b) annees & 5,8 & 5,7 & 5,4 & 6,1 & 6,9 & 6.8 & 5.9 & 6.5 & 7,0 & 6,3 & 6.2 \\
\hline$B$ annees & 7,6 & 7,3 & 7,4 & 8,1 & 0,6 & 8.2 & 7,5 & 7,9 & 8,4 & 0.3 & 7.9 \\
\hline Moyennes & 6,7 & 6.5 & 6,4 & 7,1 & 7,7 & 7,5 & 6,7 & 7.2 & 7.7 & 7,3 & \\
\hline
\end{tabular}

MOS 0.01 : pour lex moyennes des années $=0.35$ : pour les moyennes des provenances $=0.79$.

\begin{tabular}{lllllllllllll}
\hline 4 annees & 2,04 & 0,78 & 1,17 & 2,53 & 6,43 & 1,79 & $-1,29$ & 2,30 & 2,85 & 2,24 & 1,94 \\
C) annees & 2,24 & 0,80 & 1,94 & 5,44 & 5,87 & 4,12 & 2,35 & 3,42 & 5,34 & 5,16 & 3,67 \\
\hline Moyennes & 2,14 & 0.79 & 1,55 & 3,98 & 4,15 & 2,95 & 1,82 & 2,85 & 4,10 & 3,70
\end{tabular}

MCs 0.01 : pour les moyenner des années $=0,29$; pour les moyennes des provenances $=0,91$; entre le tableau $=1,29$.

\begin{tabular}{|c|c|c|c|c|c|c|c|c|c|c|c|}
\hline 4 onnéces & $\begin{array}{c}20,5 \\
(29,5)\end{array}$ & $\begin{array}{c}35,3 \\
(31,7)\end{array}$ & $\begin{array}{c}25.5 \\
(16.5)\end{array}$ & $\begin{array}{c}23,3 \\
(12,9)\end{array}$ & $\begin{array}{c}7.8 \\
(12.9)\end{array}$ & $\begin{array}{c}56.5 \\
(07,5)\end{array}$ & $\begin{array}{c}21,4 \\
(24,1)\end{array}$ & $\begin{array}{c}20,8 \\
(23,5)\end{array}$ & $\begin{array}{c}13,4 \\
(16,5)\end{array}$ & $\begin{array}{c}8.6 \\
(14.1)\end{array}$ & $\begin{array}{c}21,3 \\
(22,8)\end{array}$ \\
\hline \multicolumn{12}{|c|}{ 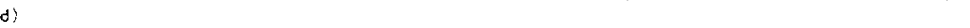 } \\
\hline 8 arnét & $\begin{array}{c}25,0 \\
(26,3)\end{array}$ & $\begin{array}{c}24.3 \\
(25.0)\end{array}$ & $\begin{array}{c}6.4 \\
(10,2)\end{array}$ & $\begin{array}{c}28 \\
(7,8)\end{array}$ & $\begin{array}{c}1,6 \\
(4,6)\end{array}$ & $\begin{array}{c}17,2 \\
(21,2)\end{array}$ & $\begin{array}{c}126 \\
(15,8)\end{array}$ & $\begin{array}{c}9,1 \\
(14,3)\end{array}$ & $\begin{array}{c}0,2 \\
(0,8)\end{array}$ & $\begin{array}{c}2,7 \\
(7,7)\end{array}$ & $\begin{array}{c}10,2 \\
(13,4)\end{array}$ \\
\hline Koy ennes & $\begin{array}{c}22,0 \\
(24,9)\end{array}$ & $\begin{array}{c}29,8 \\
(28,3)\end{array}$ & $\begin{array}{c}21,0 \\
(13,3)\end{array}$ & $\begin{array}{c}8.0 \\
(12.5)\end{array}$ & $\begin{array}{c}4,7 \\
(8,8)\end{array}$ & $\begin{array}{c}36,9 \\
(34,3)\end{array}$ & $\begin{array}{c}17.0 \\
(19,9)\end{array}$ & $\begin{array}{c}14,9 \\
(18,9)\end{array}$ & $\begin{array}{c}6,7 \\
(0,6)\end{array}$ & $\begin{array}{c}5.7 \\
(10.9)\end{array}$ & \\
\hline
\end{tabular}

MDS 0.01 : des tranfornations angliaires en parentheses: pour les moyennes des annees $=4,01$; pour les moyennes des provenances $=12.7$

\begin{tabular}{rrrrrrrrrrrrr}
\hline 4 années & 845 & 764 & 771 & 817 & 832 & 770 & 826 & 841 & 790 & 808 & 806 \\
8 annees & 994 & 906 & 929 & 1072 & 1050 & 997 & 959 & 939 & 1058 & 1043 & 994 \\
\hline Moyennes & 914 & 834 & 850 & 944 & 941 & 884 & 892 & 890 & 924 & 925
\end{tabular}

mDS 0,01 ; pour les moyennes des années $=23,0$; pour les moyennes des provenances $=72,9$.

\begin{tabular}{lllllllllllll}
\hline f) années & 538 & 501 & 498 & 532 & 538 & 509 & 533 & 537 & 502 & 515 & 520 \\
$\qquad$ années & 649 & 510 & 609 & 683 & 686 & 657 & 638 & 627 & 690 & 685 & 654 \\
\hline Moyennes & 593 & 556 & 554 & 610 & 612 & 583 & 586 & 582 & 596 & 600
\end{tabular}

MDS 0,01 : pour lee moyenres des années $=17,2$ : pour les moyennes des provenances $=38,4$.

\begin{tabular}{lllllllllllll}
\hline 4 années & 8.9 & 8,1 & 8,6 & 8,3 & 8,9 & 7,8 & 8,1 & 8,2 & 9,1 & 7,9 & 8,4 \\
(2) années & 10,5 & 10,1 & 10,8 & 11,3 & 11,2 & 10,2 & 10,8 & 10,0 & 11,3 & 10,7 & 10,7 \\
\hline Moyennes & 9,7 & 9,1 & 9,7 & 9,8 & 10,0 & 9,0 & 9,4 & 9,1 & 10,2 & 9,3 &
\end{tabular}

MDS 0,01 : pour les moyennes des années $=0,35$; pour les moyennes des provenances $=0,79$. 


\subsection{Largeur et hauteur des aiguilles}

Les valeurs moyennes de ces deux caractères sont assez homogènes sur les aiguilles des jeunes plantes (pour la seule largeur quelques différences peuvent être mises en évidence, tabl. 2), tandis que sur les arbres adultes, parallèlement à une augmentation généralisée des dimensions (tabl. 3), l'on peut relever des différences très évidentes entre les provenances grecques et celles de l'Italie méridionale d'un côté, et les provenances A 17, A 25, A 29 et A 30, de l'autre (tabl. 4). La classification reste la même, aussi bien pour la hauteur que pour la largeur, et, si l'on considère l'allure déjà observée pour les aiguilles des jeunes plantes, il paraît évident que les provenances grecques ont des dimensions décidément réduites par rapport à celles qui sont plus occidentales, ou «d'altitude".

\subsection{Nombre moyen et position des canaux sécréteurs}

Le nombre moyen des canaux sécréteurs (assez petit dans les jeunes plantes) diffère significativement entre les provenances, aussi bien dans les jeunes plantes que dans les cas des arbres adultes (tabl. 2, 3). Les jeunes plantes des provenances occidentales et/ou «d'altitude» (A 17, A 25, A 29, A 30) ont des canaux sécréteurs en plus grand nombre par rapport aux provenances grecques (A 3 et A 4), et d'Italie du Sud, bien que, avec ces dernières, la différence soit moins évidente ; à l'état adulte les différences sont encore plus fortes et l'augmentation des canaux sécréteurs avec l'âge est plus évidente dans les provenances qui en avaient déjà davantage en tant que jeunes plantes (tabl. 3, 4).

La situation est moins claire en ce qui concerne la position des canaux sécréteurs, surtout pour les jeunes plantes (tabl. 2) ; sur les arbres adultes on observe une tendance à la réduction des canaux sécréteurs en position médiane (tabl. 3). Pour les provenances occidentales la réduction entraîne des valeurs très basses (moins 3 p. 100 sur les plantes adultes, et 10 p. 100 sur la moyenne des deux années), tandis que pour les provenances orientales une telle réduction ne se présente pas (A 2) ou bien elle est très limitée (A 3) (tabl. 4); on observe la réduction la plus évidente en A 26, là où les canaux en position médiane baissent de 56 p. 100 à 17 p. 100.

\subsection{Nombre de lignes stomatifères}

Sur les jeunes plantes le nombre total des lignes stomatifères est assez homogène ; quelques différences existent toutefois en ce qui concerne la distribution sur le côté dorsal et ventral; ce dernier en particulier présente des différences parmi les provenances (A 26 et A 30 diffèrent de A 3, A 4 et A 29) (tabl. 2). Les différences deviennent encore plus évidentes sur les arbres adultes : parmi les provenances aussi bien le nombre total, que la distribution des lignes sur le côté dorsal et ventral diffèrent significativement (tabl. 3).

Sur le côté ventral l'ordre des provenances déjà établi pour les jeunes plantes se maintient : les provenances grecques A 3 et A 4 en présentent un plus grand nombre. Sur le côté dorsal et en totalité, les mêmes provenances, ajoutées à A 28 , en ont le nombre plus réduit. Pour ce caractère également l'on observe une augmentation, généralisée à toutes les provenances, lorsque l'on passe des jeunes plantes aux arbres adultes. 


\subsection{Nombre de stomates par centimètre}

Le nombre de stomates par centimètre diffère significativement selon les provenances : les provenances grecques A 2, A 3 et A 4, par rapport aux autres (tabl. 3) en ont un nombre plus élevé.

\subsection{Epaisseur de l'hypoderme et nombre de cellules hypodermiques}

Ces deux caractères anatomiques sont semblables pour toutes les provenances.

\subsection{Epaisseur de la cuticule}

La paroi des cellules épidermiques de Pinus halepensis n'apparaît pas cutinisée et l'on observe une seule couche cuticulaire déposée en dehors de la paroi. Quelquefois la cutine semble imprégner la lamelle moyenne, en englobant complètement la cellule. L'épaisseur de la cuticule diffère significativement selon les provenances (tabl. 3) : A 17, A 29 et A 30 (provenances "d'altitude») ont une cuticule plus épaisse que celle d'A 3.

\section{Discussion}

Les données ont mis en évidence des différences considérables concernant les caractères structuraux et anatomiques des aiguilles selon les provenances de Pinus halepensis Mill. examinées au cours de cette recherche.

En ce qui concerne les caractères étudiés, les différences les pius remarquables ont été mises en évidence sur les aiguilles des arbres adultes, tandis que, pour les jeunes plantes, seuls quelques caractères en présentent.

De plus, toujours sur les arbres adultes, de fortes corrélations ont été trouvées entre tous les caractères des aiguilles et quelques caractéristiques (latitude, longitude, et altitude) des régions dont proviennent les semences, ainsj qu'entre les caractères cux-mêmes (tabl. 5).

La longueur de l'aiguille et celle de la gaine sont des caractères dont la valeur taxinomique est très discutée : MERGEN et al. (1965) remarquent que pour ces caractères une ample variabilité existe dans l'espèce, et on peut impliquer une forte influence du milieu. Toutefois Mergen lui-même (1963), Gellini (1968), LeE (1968) et Coláne (1971), ont trouvé que des différences significatives existent entre la longueur des aiguilles des plantes cultivées dans leur propre milieu d'origine et celle des plantes qui proviennent d'autres régions. Les données de cette recherche semblent confirmer la variabilité de ces caractères; il est toutefois remarquable qu'un bon accord existe avec les valeurs trouvées par ColAONE (1971) sur deux provenances italiennes (Terni et Taranto) assimilables à A 26 et A 27, utilisées dans notre recherche ( 80 et $86 \mathrm{~mm}$ par rapport à 80,4 et $85,7 \mathrm{~mm}$ ), et que les petites différences observées soient dues soit à l'âge des arbres (15 et 8 ans) soit aux régions de croissance (Cecina et Arezzo). La longueur des aiguilles, bien qu'elle ne soit pas significativement différente selon les provenances, est cependant le seul caractère qui soit en corrélation avec la latitude (tabl. 5); ce résultat est également signalé par Mergen (1963) sur Pinus strobus et par Colaone (1971) sur Pinus pinaster. 


\section{TABLEAU 5}

Matrice de corrélation : Pinus halepensis Mill.

Correlation matrix : Pinus halepensis Mill.

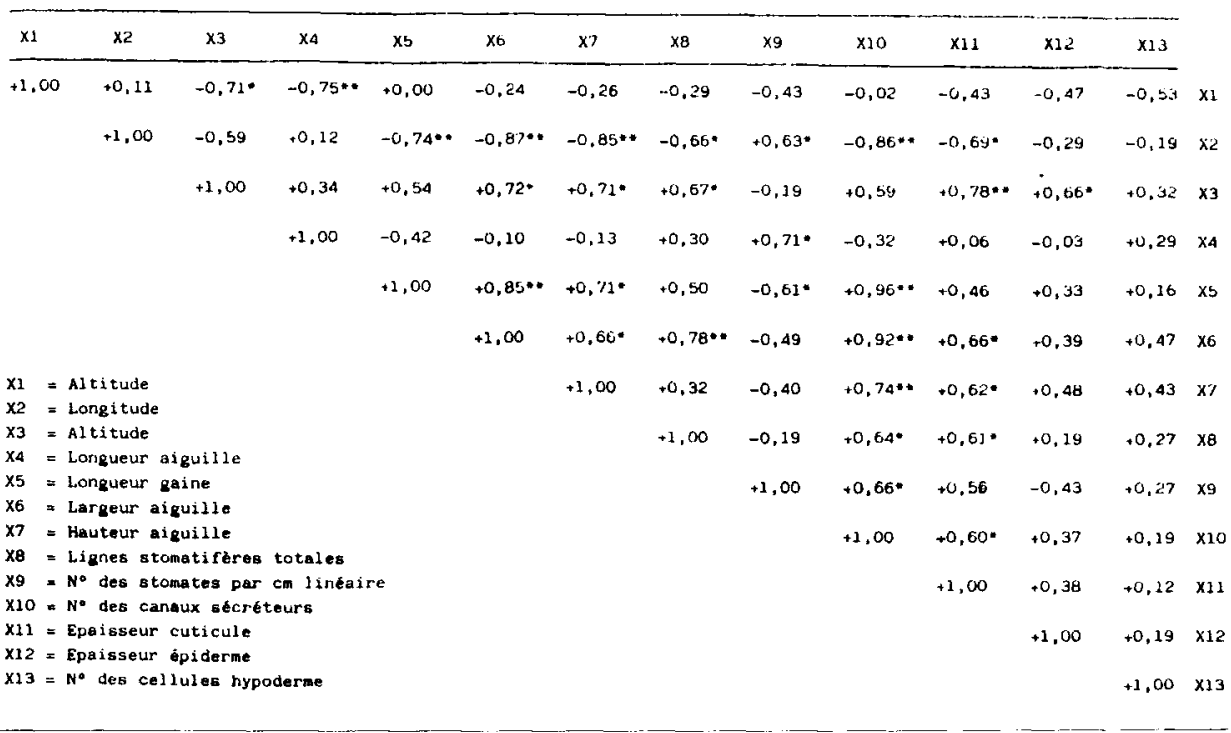

Selon DE WALL (1940), la longueur de la gaine est un caractère qui n'est influencé ni par le milieu ni par l'âge de la plante, observation non confirmée par notre recherche (tabl. 4). La longueur de la gaine des provenances grecques (A 2, A 3 et A 4) est plus élevée ( $7 \mathrm{~mm}$ ou plus, contre $4,5 \mathrm{~mm}$ ) par rapport aux valeurs reportées par Panetsos (1975) sur des populations de Pinus halepensis qui proviennent de régions très proches de celles que nous avons considérées.

Largeur et longueur de l'aiguille diffèrent selon les provenances sur les arbres adultes. Les valeurs observées s'accordent avec ce que CoLAONE (1971) a constaté, et, dans le cas de A 26, la correspondance est parfaite avec sa provenance «Terni» ( $\mathrm{L}=0,997 \mathrm{~mm}$ et $\mathrm{H}=0,657 \mathrm{~mm}$ contre $\mathrm{L}=1,0 \mathrm{~mm}$ et $\mathrm{H}=0,65 \mathrm{~mm}$ ). Stover (1944), pour Pinus contorta, trouve que les dimensions des aiguilles des plantes de milieu moyennement humide sont plus grandes que celles de milieu xérique; cette considération paraît s'accorder à notre cas, puisque les plantes de provenance grecque (A 3 en particulier), avec les aiguilles les plus réduites (tabl. 4), sont en fait originaires d'un milieu plus xérique que les autres (Walter \& Lieth, 1967). De plus, pour ce caractère, une double corrélation a été mise en évidence : de signe négatif avec la longitude et de signe positif avec l'altitude (tabl. 5).

Le nombre de canaux sécréteurs est un caractère dont la valeur taxinomique est à débattre, étant donnée sa grande variabilité (Harlow, 1947 ; White \& BEals, 1962 ; Gellini, 1968) : au point que Roller (1966) soutient la nécessité de spécifier 
l'âge de la plante (ou le stade de développement), l'âge de l'aiguille et la position dans la cime.

Les résultats de notre recherche confirment ce que WhITE \& BEALS (1962) ont trouvé sur la liaison entre nombre des canaux secréteurs et l'âge de la plante; de plus ils montrent l'influcnce du milieu (le nombre moyen des canaux sécréteurs des provenances A 2, A 3 et A 4 est nettement inférieur à celui que PANETsos (1975) a trouvé sur des populations grecques de $P$. halepensis); mais ils confirment aussi une variation probable dans l'espèce liée à la longitude, ainsi que Lee (1968) l'a démontré pour Pinus nigra et Colaone (1971) pour Pinus halepensis. Pour ce caractère également, un parfait accord existe entre la provenance de «Terni» et A $26: 4,3$ contre 4,1 .

La position des canaux sécréteurs est considérée par quelques auteurs (Dor \& MoriKaWA, 1929 ; Hartow, 1947) comme importante pour l'identification des pins, tandis que d'autres (Rollek, 1966) jugent ce critère surestimé. Les provenances grecques et d'Italie du Sud que nous avons considérées présentent le pourcentage le plus élevé de canaux sécréteurs en position médiane; cette situation, ajoutée au petit nombre de canaux sécréteurs présents dans ces provenances, peut conduire à les différencier des provenances occidentales.

Le nombre total des lignes stomatifères est en corrélation négative avec la longitude et positive avec l'altitude (tabl. 5), parallèlement à ce qui a été mis en évidence pour la largeur et la hauteur des aiguilles.

Arbez \& Millier (1971) estiment ce caractère parmi les meilleurs pour discriminer les sous-espèces en Pinus nigra; LEE (1968) considère ce caractère relativement indépendant du milieu, et il trouve une relation entre le nombre total des lignes stomatifères et les dimensions des aiguilles. D’après nos données il en est de même (tabl. 5), toutefois il faut noter que les provenances grecques, quoique caractérisées par une largeur des aiguilles inférieure aux autres, ont cependant un nombre de lignes stomatifères supérieur sur le côté ventral (tabl. 2, 3).

Le nombre de stomates par unité de longueur est en corrélation posilive avec la longitude, étant par conséquent de signe opposé à la corrélation trouvée pour les lignes stomatifères. Une étude de Mergen (1958) sur des provenances de Pinus elliottii indique, pour le nombre de stomates par unité de longueur, une tendance semblable à celle que nouts avons trouvée, tandis que, au cours d'un travail ultérieur (1963) sur Pinus sirobus un gradient nord-sud est mis en évidence. Vibakovic (1958, 1966), Keng \& LitTle (1961) et Mergen (1965) considèrent lc nombre de stomates comme un caractère génétiquement stable et donc utilisable pour discriminer les lybrides; de la même façon GAUSSEN (1960) l'avait utilisé pour reconnaître les provenances et les sous-espèces.

L'épaisseur de la cuticule, plus consistante dans les provenances A 17, A 29 et A 30, se trouve en corrélation avec la longitude (négativement) et avec l'altitude (positivement) (tab1. 5). Selon Stover (1944), Baig \& Tranoulllini (1976) et Kovalev (F.A. 4838 , 1981), au moins jusqu'aux régions-limites pour la végétation, c’est précisément avec l'altitude que l'épaisseur de la cuticule est en meilleure corrélation. Il faut souligner non sculement qu'une très bonne corrélation avec l'altitude a été mise en évidence au cours de notre recherche, mais ajoutons aussi que l'on trouve dans les régions occidentales $P$. halepensis jusqu'à des altitudes de $1500-2000 \mathrm{~m}$ 


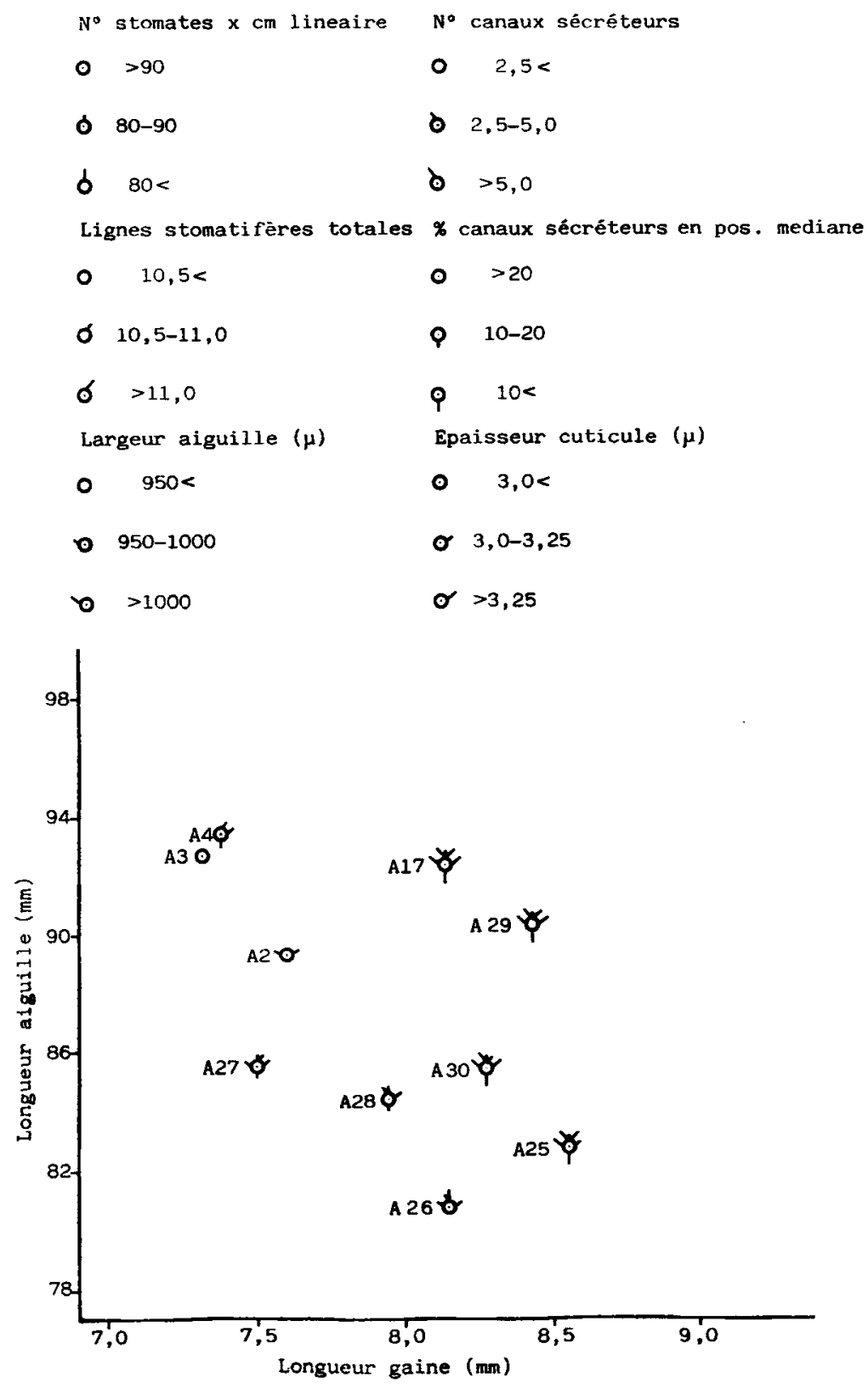

FIG. 1

Populations de Pinus halepensis Mill.

Populations of Pinus halepzass.s Mill. 
(au Maroc), par contre, dans les régions orientales il a tendance à disparaître à partir de $200-300 \mathrm{~m}$.

Le nombre des couches de cellules de l'hypoderme est un caractère dont la crédibilité est controversée. Vidakovic (1958), Gelinini (1968) et LeE (1968) soutiennent qu'il ne se ressent pas de l'influence du milieu, ayant donc une remarquable importance pour la classification des provenances, tandis que d'un avis différent sont Schutt \& Hattemer (1958) et Kriebel \& Flower (1955). D'après nos données il ne nous paraît pas être un caractère suffisant pour discriminer les provenances.

Des considérations analogues peuvent être faites à l'égard de l'épaisseur des cellules épidermiques, même si le caractère est en corrélation avec l'altitude. LARSEN (1927), pour Pinus contorla, estime que l'épaisseur de l'épiderme peut représenter, dans quelques emplacements, une adaptation à des conditions xériques, tandis que CRitchfield (1957), toujours sur Pints contorta, ne trouve aucune corrélation entre ce caractère et les régions d'où proviennent les semences, bien qu'elles soient très différentes du point de vue climatique.

\section{Conclusions}

Les connaissances sur la variation de quelques caractères structuraux et anatomiques des aiguilles, s'étendent à Pinus halepensis Mill. grâce à cette recherche. De telles variations avaient déjà été observées sur d'autres espèces du genre Pinus, associées à l'âge des plantes, se manifestant également durant des laps de temps parfois très courts. De la même façon nous avons observé que les caractères qui permettent de distinguer les provenances lorsque les plantes sont jeunes, ne sont pas les mêmes une fois celles-ci devenues adultes. De plus, parmi ces caractères, deux seulement (nombre de canaux sécréteurs et nombre de lignes stomatifères du côté ventral de l'aiguille) maintiennent leur stabilité, même lorsque leurs valeurs absolues changent.

D'autre part les caractères qui étaient homogènes sur les jeunes plantes (largeur et hauteur de l'aiguille, nombre de lignes stomatifères totales et sur le côté dorsal) sont devenus des caractères discriminants pour les arbres adultes.

Le milieu paraît exercer une influence sensible sur quelques caractères morphologiques et anatomiques. D'après la comparaison entre nos résultats et ceux de Colaone (1971) sur des populations de Pinus halepensis différentes quant à leur lieu dorigine, la seule provenance « Terni » présente avec évidence des valeurs (longueur, largeur, hauteur de l'aiguille et nombre de canaux sécréteurs) qui s'accordent avec ce que l'on a trouvé sur A 26 (qui provient de la même région, et qui était cultivée, dans les deux cas, tout près de son lieu d'origine); par contre les différences sont considérables en ce qui concerne les autres provenances. Quelques caractères (longueur, largeur et hauteur de l'aiguille, longueur de la gaine, nombre de canaux sécréteurs) ont montré des différences encore plus évidentes entre nos provenances A 3 et A 4, et les populations grecques étudiées pai PANETsos (1975) en Grèce; bien que provenant de régions très proches, le milieu de croissance, substantiellement différent, a joué un rôle très important évident. 


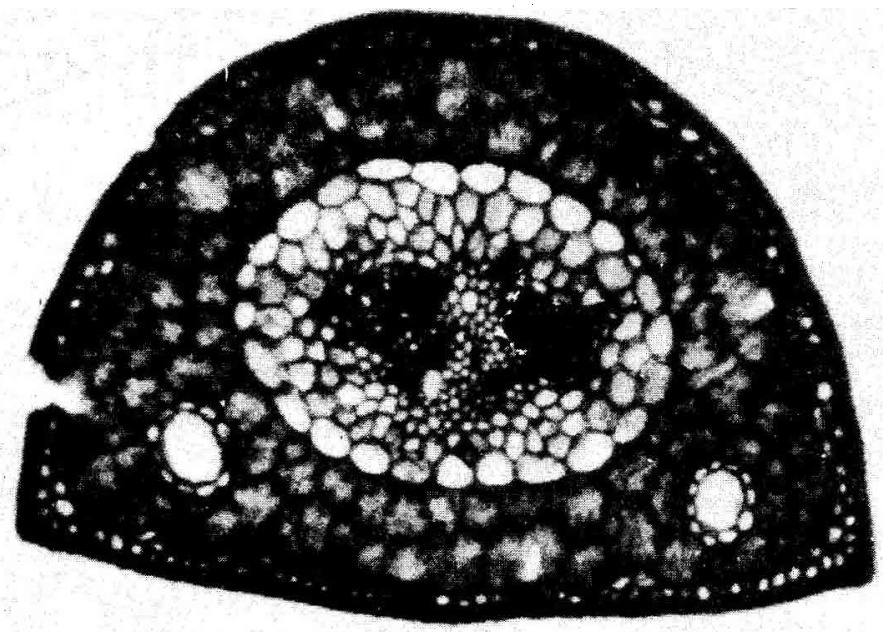

FIG. 2

Coupe transversale de l'aiguille d'une plante de la provenance A 2 (représentative des provenances grecques).

Transverse section of a needle from a tree of the provenances $A 2$ (representative of the Greek provenances).

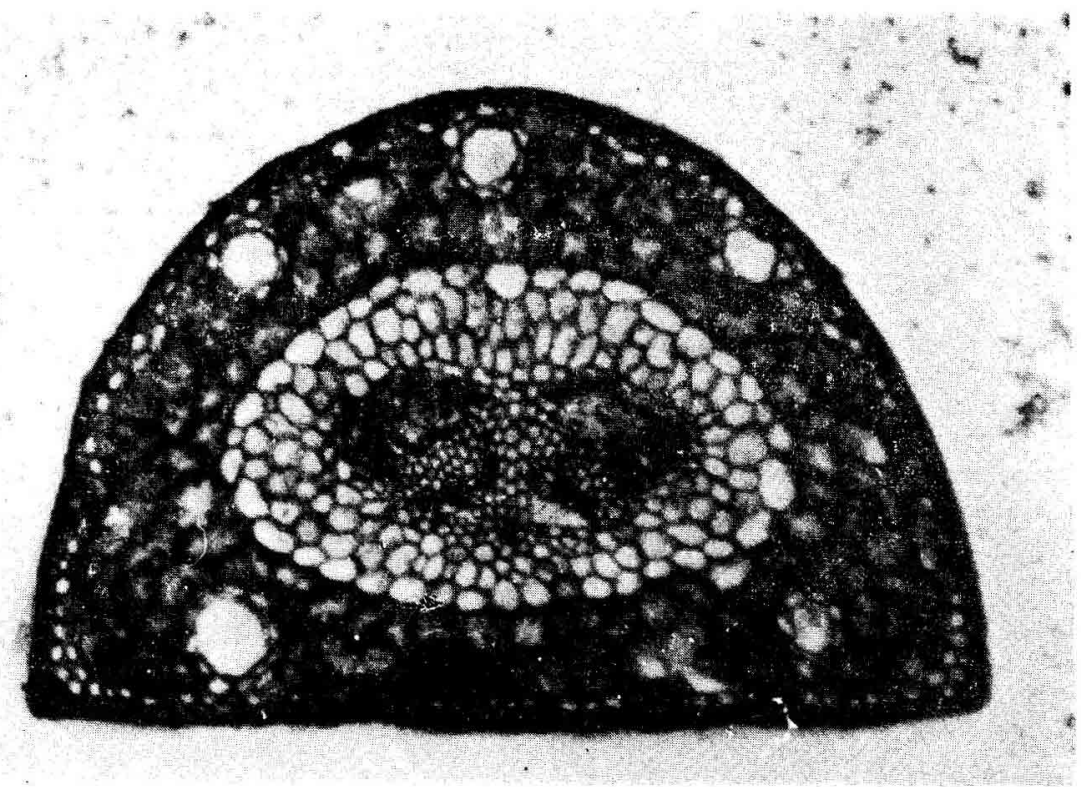

Fig. 3

Coupe transversale de l'aiguille d'une plante de la provenance A 27 (représentative des provenances de l'Italie méridionale).

Transverse section of a needle from a tree of the provenance $A^{4} 27$ (representative of the south Italian provenances). 


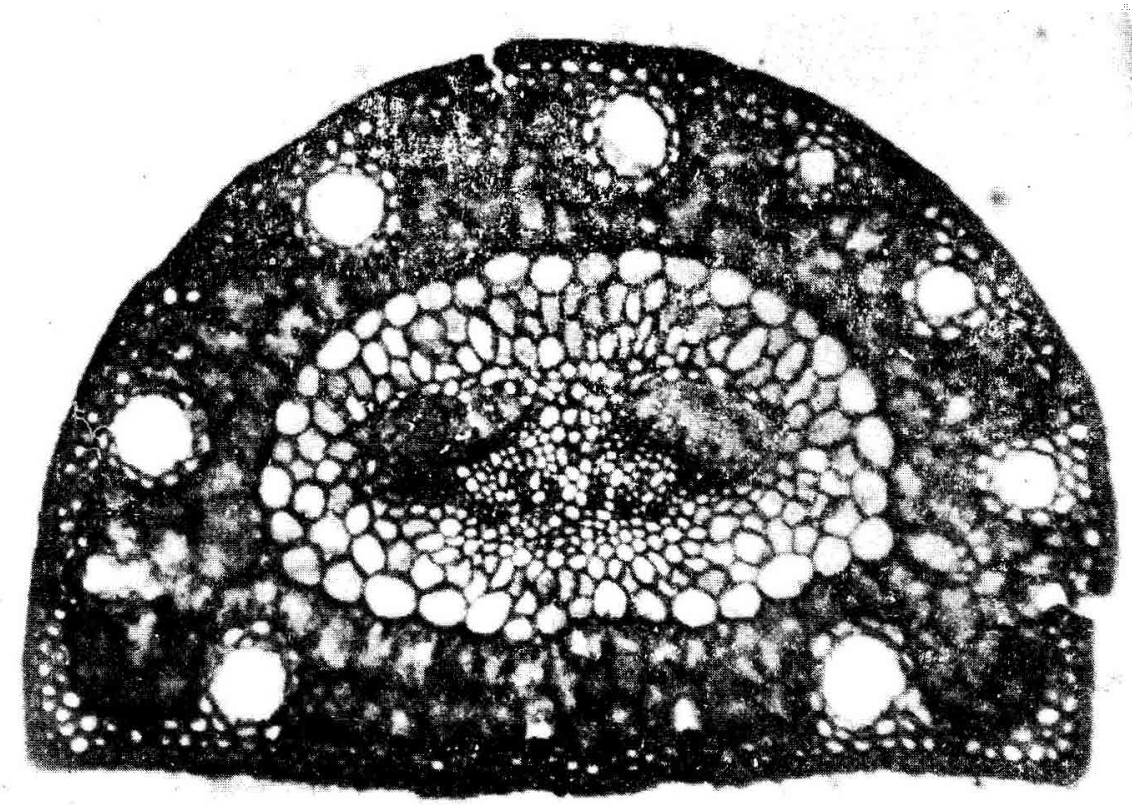

FIG. 4

Coupe transversale de l'aiguille d'une plante de la provenance A 29 (représentative des provenances occidentales et/ou «d'altitude»).

Transverse section of a needle from a tree of the provenance $A 29$ (representative of the western and/or « of altitude» provenances).

Compte tenu de ces considérations, nos résultats permettent, grâce à la stabilité de certains caractères et leurs associations sur quelques populations, de distinguer parmi les provenances de Pinus halepensis Mill. des groupements bien différenciés (fig. 5).

Les provenances grecques A 3 et A 4 (et en moindre mesure A 2) diffèrent (fig. 2), des provenances occidentales (et/ou «d'altitude») A 17, A 25, A 29 et A 30 (fig. 3) pour la quasi totalité des caractères, tandis que les provenances d'Italic centreméridionale A 26, A 27 et A 28 (fig. 4) se placent en position intermédiaire, ressemblent à l'un ou à l'autre groupe, selon le caractère considéré. La figure 1, où les caractères sont représentés selon la méthode d'ANDERson (1953) "pictorialized scatter diagram », méthode généralement utilisée pour la discrimination des hybrides, fait très bien ressortir ce résultat.

L'analyse des régressions, qui met en évidence la variation ouest-est de tous les caractères examinés (avec comme seule exception la longueur de l'aiguille), confirme l'existence d'une variation géographique en bon accord avec les groupements de provenance déjà mentionnés (fig. 5).

L'altitude à laquelle sont liés quelques-uns des caractères observés, représente également un composant non indifférent pour la corrélation avec la latitude (tabl. 5). 


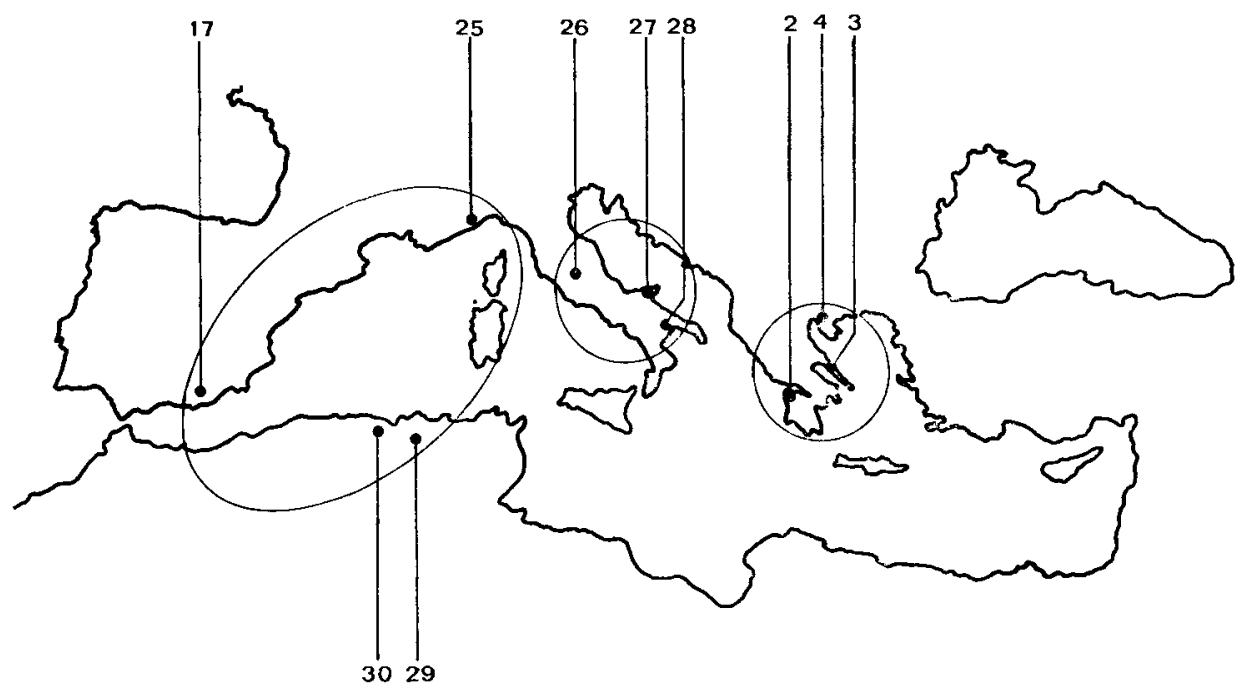

FIG. 5

Emplacement des provenances de Pinus halepensis Mill. et regroupements proposés.

Location of Pinus halepensis Mill. provenances and groupings proposed.

La cuticule a tendance à augmenter, selon quelques auteurs, jusqu'à certaines limites au-dessus desquelles la tendance se renverse, et l'on observe une nette réduction (Baig \& Tranquillini, 1976, ne trouvent aucune différence sur des plantes de Picea abies poussant entre 1000 et $1940 \mathrm{~m}$ ); de plus, en milieu moyennement humide, les dimensions de l'aiguille augmentent par rapport à ce que l'on trouve en milieu xérique. Pinus halepensis uniquement dans les stations les plus méridionales et occidentales trouve un milieu au climat plus favorable, et il peut y coloniser des altitudes plus élevées.

En ce qui concerne d'autres caractères et comportements, l'existence de différences parmi la population de $P$. halepensis a été démontrée au cours d'études préalables (Calamassi et al., 1980 ; Calamassi, 1982 ; Falusi et al., 1983 ; Calamassi et al., 1984), là où la présence de variations écotypiques pour de nombreux caractères et comportements en Pinus halepensis Mill. soutenue par plusieurs auteurs (NAHAL, 1962 ; Ferre, 1965 ; Debazac \& Tomassone, 1965 ; Colaone, 1971 ; Palmberg, 1975) est vérifiée avec certitude.

Pour quelques-uns des caractères observés (nombre de stomates par $\mathrm{cm}$ et nombre de lignes stomatifères en position ventrale) il semble très probable qu'une forte liaison existe avec la résistance à l'aridité. Un nombre élevé de stomates est généralement considéré comme une adaptation xéromorphique, et les provenances grecques A 2, A 3 et A 4, qui présentent les valeurs les plus élevées, ont résisté le mieux aux stress hydriques pendant la germination et les premières phases de la croissance de la racine (CAlamassi et al., 1980; Falusi et al., 1983) : elles proviennent en effet d'un milieu au climat thermo-méditerranéen avec 100-150 jours secs par an (WALTER \& LiETh, 1967). Un bon accord existe pour d'autres provenances également : 
A 29, qui paraissait être relativement résistante au sec, porte un nombre de stumates inférieur à celui des provenances grecques seulement (tabl. 3) ; tandis que A 26, très sensible aux stress hydriques, présente le nombre de stomates le plus réduit parmi les provenances examinées.

Reçu le 5 août 1985.

Accepté le 5 février 1986.

\section{Summary \\ Identification of provenances of Pinus halepensis Mill. on the basis of the anatomical and morphological neddle structures}

Anatomical and morphological structures of needles in seedlings and adult plants of 10 provenances of Pinus halepensis Mill., have been analyzed in order to characterize their provenances and to ascertain the discriminating characteristics as a function of growth stage and the environment.

There is evidence of a remarkable variability in some characteristics related to the age and to the environmental conditions during the plant growth, in fact it appears that the characteristics which can be chosen to discriminate between plants from the different provenances do not maintain this ability from seedling to the adult stage.

Nevertheless some characters are constant and grouped in some populations, therefore allowing the discrimination of some well differenciated groups : the Greek provenances (A 2, A 3, A 4) on one side, and the western or «of altitude 》 (A 17, A 25, A 29, A 30) on the other, meanwhile the south-central Italian provenances (A 26, A 27, A 28) are situated in a central position between these two groups.

A geographical variation is also in evidence, showing a west-east trend.

\section{Références bibliographiques}

ANDERSON E., 1953. Introgressive hybridization. Biol. Rev., 28, 280-307.

Arbl:z M., Millier C., 1971. Contribution à l'étude de la variabilité géographique de Pinus nigra Arn. Ann. Sci. For., 28, 23-49.

Baig M.N., Tranquituini W., 1976. Studies on upper timberline : morphology and anatomy of Norway spruce (Picea abies) and stone pine (Pinus cembra) needles from various habitat conditions. Can. J. Bot., 54, 1622-1632.

Calamassi R., 1982. Effetti della luce e della temperatura sulla germinazione dei semi in provenienze di Pinus halepensis Mill. e Pinus brutia Ten. It. For. Mont., XXXVII, 174-187.

Calamassi R., Falusi M., Tocci A., 1980. Variazione geografica e resistenza a stress idrici in semi di Pinus halepensis Mill., Pinus brutia Ten., Pinus eldarica Medw. Ann. Ist. Sper. Selv. Arezzo, XI, 195-230.

Calamassi R., Falusi M., Tocci A., 1984. Effets de la température de germination et de la stratification sur la germination des semences de Pinus halepensis Mill. Silv. Genet., 33, $133-139$.

Recherche soutenue par l'Université de Florence avec la contribution du Ministère de l'Instruction Publique pour la Recherche Scientifique. 
ColaOne M., 1971. Indagini preliminari sui caratteri anatomici degli aghi di alcune provenienze di Pinus Pinaster Ait. e Pinus halepensis Mill. It. For. Mont., XXVI, 164-171.

Critchfield W.B., 1957. Geographic variation in Pinus contorta. Maria Moors Cabot Foundation, Publ. 3, 118 p. Cité par Mergen F., 1963.

Debazac E., Tomassone R., 1965. Contribution à une étude comparée de Pins méditerranéen de la section halepensis. Ann. Eco. Natio. des Eaux et For., 27, 215-256.

Dor T., Morikawa K., 1929. An anatomical study of leaves of the genus Pinus. J. Dept. Agric., Kjushu University, II, N“ 6, 149-168. Cité par LEE C.H., 1968.

Durrell L.W., 1916. Notes on some North conifers based on leaf characters. Iowa Acad. Sci., 23, $519-582$.

De Wall W.B., 1940. A diagnostic taxonomic costant for separating slash and longleaf pines. Fla. Acad. Sci. Proc., 4, 113-115.

Evans L.S., Miller P.R. ,1972. Ozone damage to ponderosa pine : an istological and Hystochemical appraisal. Amer. J. Bot., 59, 297-304.

Falusi M., Calamassi R., Tocci A., 1983. Sensitivity of seed germination and scedling root growth to moisture stress in four provenances of Pinus halepensis Mill. Silv. Genet., 32, 4-9.

Falusi M., Calamassi R., Tocci A., 1984. Resistenza al freddo in Pinus halepensis Mill. Pinus brutia Ten. e Pinus eldarica Medw. Atti Soc. Tosc. Sci. Nat., Mem., Serie B, $\mathrm{XCl}, 1-23$.

Ferre Y. DE, 1965. Structure des plantules et systématique du genre Pinus. Bull. Soc. Hist. Nat. Toulouse, Tome 100, Fasc. 1-2.

Flora Europaea, 1964. Lycopodiaceae to Platanaceae. Vol. 1, 35. Cambridge Univ. Press.

Francini E., 1953. Il pino d'Aleppo in Puglia. Ann. Fac. Agraria, Bari, VIII, 309-416.

Gaussen H., 1960. Les Gymnospermes actuelles et fossiles. Toulouse, Fasc. VI, 14-29.

Gellini R., 1968. Posizione sistematica del Pino nero di Villetta Barrea in base ai caratteri anatomici degli aghi. Ann. Accad. It. Sci. For., 17, 101-122.

Harlow W.M., 1947. The identification of the pines of the United States native and introduced by needle structure. Bull. New, York Coll. For., Syracuse Univ., XX, Fasc. 2.

Hosie R.C., 1969. Native trees of Canada. Can. for. Service Departement of Environment, Ottawa.

JARIG M., 1962. Beiträge zur Nadelanatomie und Taxonomie der Gattung Pinus L. Willdonovia, 3, 329-366.

Keng H., Lirtle E.L. Jr., 1961. Needle characteristics of hybrid pines. Silv. Genet., 10, 131-146.

Kriebel H.B., Fowler D.P., 1965. Variability in needle characteristics of soft pine species and hybrids. Silv. Genet., 14, 73-76.

LARSEN J.A., 1927. The relation of leaf structure of conifers to light and moisture. Ecology, 8, 371-377. Cité par Mergen F., 1963.

LEE C.H., 1968. Geographic variation in European black pine. Silv. Genet., 17, 165-172.

Leyton L., Juniper B.E., 1963. Cuticle structure and water relations of pines needles. Nature, 198, 770-771.

Leyton L., Armitage I.P., 1968. Cuticle structure and water relations of pines needles of Pinus radiata (D. Don). New Phytol., 67, 31-38.

Mergen F., 1958. Genetic variation in needle characteristics of slash pine and in some of the hybrids. Silv. Genet., 7, 1-9.

Mergen F., 1963. Ecotipic variation in Pinus strobus L. Ecology, 44, 716-727.

Mergen F., Stairs R.G., SNYder E.B., 1965. Natural and controlled loblolly $\times$ shortlcaf hybrids in Mississippi. For. Sci., 11, 306-314.

Mirov N.T., 1967. The genus Pinus. Ronald Press Co., New York.

Nahnl, 1., 1962. Le Pin d'Alep (Pinus halepensis Mill.). Etude taxinomique, phytogeographique, écologique et sylvicole. Ann. Eco. Natio. des Eaux et For., XIX, 208 pp. 
Nappzinn K., 1966. Anatomie des blattes. I. Gymnosperm. Enc. Plant Anatomy, Gebunde Bautragen Berlin.

Oppenheimer H.R., 1968. Drought resistance of Monterey pine needles. Isr. J. Bot., 17, $163-168$.

Palmberg C., 1975. Geographic variation and carly growth in souther-eastern semi-arid Australia of Pinus halepensis Mill, and Pinus bruta Ten. species complex. Silv. Genet., 24, $150-160$.

Panetsos K.C.P., 1981. Monograph of Pinus halepensis (Mill.) and Pinus brutia (Ten.). Ann. Forest., 9/2, 39-77.

Pelizzo A., Tocci A., 1978. Indagini preliminari su semi e semenzali di Pinus halepensis e Pinus brutia-eldarica. Ann. Ist. Sper. Selv., Arezzo, IX, 111-130.

Riva L., Vendramin G., 1983. Prime osservazioni sull'ibrido artificiale Pinus brutia $\times$ Pinus halepensis. It. For. Mont., XXXVIII, 234-248.

ROLLER K.J., 1966. Resin canal position in the needles of balsam, alpine and frasers firs. For. Sci., 12, 348-355.

STover E.L., 1944. Varying structure of conifer leaves in different habitats. Bot. Gaz., 106, $12-25$.

SchutT P., Hattamer H.H., 1958. Dic Eigung von Merkmalen der Nadelquerschnitts für Kiefern. Bastard diagnose. Silv. Genet., 8, 93-99.

Vidakovic M., 1957. Forms of Pinus nigra in Jugoslavia on the basis of anatomical structure of the needles (Yu). Glasu Sunske Pakuse, Zagreb, 13, 111-148.

VIDAkovic M., 1958. Investigations of the intermediate type between the Austrian and Scots pine. Silv. Genet., 7, 12-19.

Walter H., Lieth H., 1967. Klimadiagramm-weltatlas. G. Fischer, Jena.

Vhite D.B., BEALS H.O., 1962. Variation in number of resin canals per needles in ponderosa pine. Bot. Gaz., 124, 251-253. 